\title{
CREscimento DE Siegesbeckia orientalis SOB DIFERENTES CONDIÇões DE LUMINOSIDADE ${ }^{1}$
}

\author{
Growth of Siegesbeckia orientalis under Different Light Conditions
}

\author{
AGUILERA, D.B. ${ }^{2}$, FERREIRA, F.A. ${ }^{3}$ e CECON, P.R. ${ }^{4}$
}

\begin{abstract}
RESUMO - Neste estudo, objetivou-se contrastar os efeitos de sombreamento sobre o crescimento de $S$. orientalis, utilizando a análise de crescimento. Os experimentos foram conduzidos em vasos, a pleno sol (A) e sob sombrite 50\% (B). As plantas tiveram seus índices de crescimento determinados aos $14,28,42,56,80,94$ e 108 dias após o transplante (DAT), para A; e aos 14, 28, 42, 56, 70, 84, 108, 122 e 136 DAT, para B. O delineamento experimental foi o inteiramente casualizado, com quatro repetições. Os dados foram analisados por meio de análise de regressão. Os modelos foram escolhidos com base na significância dos coeficientes de regressão, utilizando-se o teste t até $10 \%$, no coeficiente de determinação e no fenômeno em estudo. As plantas apresentaram comportamento semelhante em A e B para a maioria das variáveis analisadas, embora as plantas sombreadas (radiação média de $218 \mu \mathrm{mol} \mathrm{m}^{-2} \mathrm{~s}^{-1}$ ) tivessem maior duração do ciclo cultural, cerca de 140 DAT, retardando os valores máximos e/ou mínimos, em relação às plantas a luz plena (média de $658 \mu \mathrm{mol} \mathrm{m}^{-2} \mathrm{~s}^{-1}$ ), com aproximadamente 110 DAT. Em B, foram obtidas também as maiores médias para a maioria dos índices avaliados - área foliar, altura, números de folhas e capítulos florais, biomassa seca total, taxa de crescimento absoluto, taxa de crescimento relativo, razão de peso foliar, razão de área foliar e área foliar específica -, sugerindo que a espécie é favorecida pelo sombreamento.
\end{abstract}

Palavras-chave: análise de crescimento, sombreamento, botão-de-ouro.

ABSTRACT - This study aimed to contrast the effects of shading on the growth of S. orientalis by applying growth analysis. The experiments were conducted in pots under full sunlight (A) and 50\% shading (B). Plant growth indices were determined at 14, 28, 42, 56, 80, 94 and 108 days after transplant (DAT) for $A$ and at 14, 28, 42, 56, 70, 84, 108, 122, 136 DAT for B. The experimental design was completely randomized, with four repetitions. Data were analyzed by means of regression analysis. The models were chosen based on the significance of the regression coefficients, using the test up to $10 \%$ in the determination coefficient and the phenomenon under study. The plants presented a similar behavior in A and B for most of the variables analyzed; however, the plants under shading (mean radiation of $218 \mu \mathrm{mol} \mathrm{m} \mathrm{m}^{2} \mathrm{~s}^{-1}$ had a longer cultural cycle duration (around 140 DAT) with their maximum and minimum points being much slower than the plants under full sunlight (average of $658 \mu \mathrm{mol} \mathrm{m} \mathrm{m}^{2} \mathrm{~s}^{1}$ ), with approximately 110 DAT. The plants under shading also obtained the highest averages for most of the indices evaluated, such as leaf area, height, number of leaves and floral capitulum, total dry biomass, absolute growth rate, relative growth rate, leaf weight ratio, leaf area ratio and specific leaf area, indicating that this species is favored by shading.

Key words: growth analysis, shading, shrimp-claw plant.

1 Recebido para publicação em 1.9.2003 e na forma revisada em 5.3.2004.

Parte da tese de doutorado em Fitotecnia do primeiro autor, Universidade Federal de Viçosa - UFV.

2 Doutoranda em Fitotecnia pela Universidade Federal de Viçosa - UFV; ${ }^{3}$ Professor Titular, Dep. de Fitotecnia da UFV, 36570-000, Viçosa-MG; ${ }^{4}$ Professor Adjunto, Departamento de Informática da UFV. 


\section{INTRODUÇÃO}

Siegesbeckia orientalis (botão-de-ouro) é uma espécie herbácea anual e cosmopolita, da família Asteraceae, incluída na tribo Heliantheae. No Brasil, é freqüentemente encontrada na região Sudeste, com maior ocorrência no período da primavera ao outono, em locais úmidos, sendo tolerante à iluminação difusa e podendo ser considerada daninha em algumas culturas. É também utilizada como planta medicinal (Baruah et al., 1979; Bremer, 1994; Kissmann \& Groth, 1992), sendo isolados diversos compostos, que, além de possuírem princípios ativos medicamentosos, incluem substâncias com atividade alelopática (Pudles et al., 1957; Bohlmann et al., 1979; Baruah et al., 1979, 1980; Zdero et al., 1991; Heinrich et al., 2002).

O cultivo de plantas medicinais e/ou alelopáticas envolve a possibilidade de domesticação da espécie a ser utilizada, o que implica o domínio do conhecimento dos estádios de desenvolvimento. A estratégia de obtenção de biomassa, assim como de seus princípios ativos, requer conhecimento sobre a forma de propagação, adaptação ao ambiente de cultivo, forma de crescimento, senescência, etc. (Reis \& Mariot, 2002).

A análise de crescimento ainda é o meio mais simples e preciso para inferir a contribuição de diferentes processos fisiológicos para o crescimento vegetal, sendo útil no estudo de variações entre plantas geneticamente diferentes ou sob diferentes condições ambientais. Mediante a análise de crescimento, é possível conhecer a cinética de produção de biomassa das plantas, sua distribuição e eficiência ao longo da ontogenia (Benincasa, 1988).

Estudos de crescimento de plantas têm sido amplamente utilizados para o conhecimento da ecofisiologia de diferentes espécies submetidas a diferentes condições de luminosidade, seja pelo controle da radiação incidente, por meio do emprego de lâmpadas de diferentes comprimentos de ondas ou câmaras de crescimento (Cooper, 1967; Jeangros \& Nosberger, 1992; Cooley et al., 2000; Musil et al., 2002), ou pelo uso de telados ou plásticos redutores da radiação (Vlahos et al., 1991; Dale \& Causton, 1992a, b, c; Pushpakumari \& Sasidhar, 1992;
Sharma, 1994; Muroya et al., 1997; Farias et al., 1997).

Objetivou-se, neste estudo, contrastar os efeitos de sombreamento sobre o crescimento de S. orientalis, utilizando-se de técnicas de análise de crescimento.

\section{MATERIAL E MÉTODOS}

O experimento foi conduzido de agosto de 2002 a janeiro de 2003, em casa de vegetação na Universidade Federal de Viçosa (UFV), Viçosa - MG, localizada a $20^{\circ} 45^{\prime}$ S, $46^{\circ} 51^{\prime} \mathrm{W}$ e com altitude média de 689 metros. Os aquênios de Siegesbeckia orientalis foram coletados de plantas nascidas espontaneamente no campus, em março, e armazenados por cinco meses em sacos de papel kraft, em local seco e arejado, sob temperatura ambiente.

A semeadura foi feita em bandejas de isopor, preenchidas com substrato para formação de mudas e mantidas em casa de vegetação, sem que a água fosse fator limitante. Mudas com $8 \mathrm{~cm}$ de altura foram transplantadas 30 dias após a semeadura para local sombreado, sob sombrite - 50\% (baixa radiação incidente - B) e a pleno sol (A). As unidades experimentais foram constituídas por vasos de $3 \mathrm{~kg}$, contendo uma planta cada, preenchido com solo (Oxissolo/LVA), onde previamente realizou-se uma adubação de nivelamento (NPK $-4: 14: 8$ ), mais $25 \%$ de composto, sem que fosse necessária a realização de calagem. As plantas foram colhidas a intervalos de 14 dias e seus indices de crescimento determinados aos 14 , $28,42,56,80,94$ e 108 dias após o transplante (DAT), para o experimento A; e aos 14, 28, 42, $56,70,84,108,122$ e 136 DAT, para o B. O delineamento experimental foi o inteiramente casualizado, com quatro repetições. Os dados foram submetidos à análise de regressão. Os modelos foram escolhidos com base na significância dos coeficientes de regressão, utilizando-se o teste t e adotando-se o nível de até $10 \%$ de probabilidade, no coeficiente de determinação $\left(\mathrm{R}^{2}=\right.$ S.Q.Regressão/S.Q.Luminosidade $)$ e no fenômeno em estudo.

Semanalmente, foi modificada a posição dos vasos e efetuou-se capina manual. A radiação incidente média nas duas condições de luminosidade foi medida com o auxílio de 
um fotômetro (Hansatech), modelo SKP 200, em um dia ensolarado, em seis pontos distintos do dossel e em quatro horários distintos: 7h30, $10 h, 13 h$ e $16 h$.

Foram realizadas nove e sete coletas (método destrutivo), sob sombrite e a pleno sol, respectivamente, devido à diferença na duração do ciclo de crescimento nas duas condições. Em cada coleta, foram separadas as partes das plantas: folhas (f), ramos e capítulos florais (fl) e raízes ( $r$ ), sendo determinadas as características: número de folhas e capítulos, altura do ramo principal, início da floração e senescência de folhas e área foliar $\left(A_{f}\right)$. Após a secagem das diferentes partes em estufa de renovação forçada de ar a $70{ }^{\circ} \mathrm{C}$ até massa constante, foi aferida a massa seca de raízes $(\mathrm{Wr})$, ramos e capítulos florais $\left(\mathrm{W}_{\mathrm{fl}}\right)$, folhas $\left(\mathrm{W}_{\mathrm{f}}\right)$ e total $\left(\mathrm{W}_{\mathrm{t}}\right)$.

A área foliar $\left(\mathrm{cm}^{2}\right)$ foi determinada por meio de um medidor de área foliar Li-cor, modelo LI-3100, sendo a altura medida com régua milimetrada. A partição de biomassa entre diferentes regiões da planta $\left(\mathrm{F}_{\mathrm{w}}\right)$ foi determinada em percentagem de matéria seca das diferentes partes $\left(\mathrm{W}_{\mathrm{f}}, \mathrm{W}_{\mathrm{fl}}\right.$ e $\left.\mathrm{W}_{\mathrm{r}}\right)$ em relação à matéria seca total ( $\left.\mathrm{W}_{\mathrm{t}}\right)$ ao longo do ciclo $\left(\mathrm{g} \mathrm{g}^{-1}\right)$. Com base nos resultados de massa seca acumulada e área foliar, foram também determinadas, para cada avaliação, a taxa de crescimento absoluto $\left(\mathrm{C}_{\mathrm{t}}\right)$, a taxa de crescimento relativo $\left(\mathrm{R}_{\mathrm{w}}\right)$, a taxa de assimilação líquida $\left(\mathrm{E}_{\mathrm{A}}\right)$, a razão de área foliar $\left(\mathrm{F}_{\mathrm{A}}\right)$ e a área foliar específica $\left(\mathrm{S}_{\mathrm{A}}\right)$, de acordo com Radford (1967) e Benincasa (1988).

A taxa de crescimento absoluto $\left(\mathrm{C}_{t}\right)$ dá a idéia da velocidade média de crescimento das plantas ao longo do período de observação, sendo calculada pela fórmula $\mathrm{C}_{\mathrm{t}}=\left(\mathrm{W}_{2}-\mathrm{W}_{1}\right) /\left(\mathrm{t}_{2}-\mathrm{t}_{1}\right)$, em que $\mathrm{W}_{2}$ e $\mathrm{W}_{1}$ são as massas secas de duas amostragens sucessivas e $t_{2}$ e $t_{1}$ são os dias decorridos entre as duas observações ( $\mathrm{g} \mathrm{dia} \mathrm{di}^{-1}$ ). A taxa de crescimento relativo $\left(\mathrm{R}_{\mathrm{w}}\right)$ é o aumento em gramas de massa seca por unidade de material presente num período de observação, $\mathrm{R}_{\mathrm{w}}=\left(\ln \mathrm{W}_{2}-\ln \mathrm{W}_{1}\right) /\left(\mathrm{t}_{2}-\mathrm{t}_{1}\right)\left(\mathrm{g} \mathrm{g} \mathrm{g}^{-1}\right.$ dia $\left.^{-1}\right)$. A taxa de assimilação líquida $\left(\mathrm{E}_{\mathrm{A}}\right)$ representa a taxa de fotossíntese líquida, em termos de massa seca produzida (g), por área foliar $\left(\mathrm{cm}^{2}\right)$, por unidade de tempo (dia), $\mathrm{E}_{\mathrm{A}}=\left[\left(\mathrm{W}_{2}-\mathrm{W}_{1}\right) /\left(\mathrm{A}_{2}-\mathrm{A}_{1}\right)\right] /\left[\left(\ln \mathrm{A}_{2}-\right.\right.$ $\left.\left.\ln A_{1}\right) /\left(t_{2}-t_{1}\right)\right]$, em que $A_{2}$ e $A_{1}$ são as áreas foliares de duas amostragens consecutivas. A razão de área foliar $\left(\mathrm{F}_{\mathrm{A}}\right)$ expressa a área foliar útil para a fotossintese, componente morfofisiológico, pois é a razão entre a área foliar e a massa seca total $\left(\mathrm{cm}^{2} \mathrm{~g}^{1}\right), \mathrm{F}_{\mathrm{A}}=\mathrm{A}_{\mathrm{f}} / \mathrm{W}_{\mathrm{t}}$ ou $\mathrm{F}_{\mathrm{A}}=\mathrm{S}_{\mathrm{A}} \mathrm{F}_{\mathrm{wf}}$. A área foliar específica $\left(S_{A}\right)$ é o componente morfoanatômico de $\mathrm{F}_{\mathrm{A}}$ que relaciona a superfície com a massa seca das folhas, $\mathrm{S}_{\mathrm{A}}=\mathrm{A}_{\mathrm{f}} / \mathrm{W}_{\mathrm{f}}$ $\left(\mathrm{cm}^{2} \mathrm{~g}^{-1}\right)$. A razão de massa foliar $\left(\mathrm{F}_{\mathrm{wf}}\right)$ é $\mathrm{o}$ componente fisiológico que relaciona a massa seca das folhas à massa seca total, $\mathrm{F}_{\mathrm{wf}}=\mathrm{W}_{\mathrm{f}} / \mathrm{W}_{\mathrm{t}}$ $\left(g g^{-1}\right)$.

\section{RESULTADOS E DISCUSSÃO}

As plantas de Siegesbeckia orientalis apresentaram comportamento semelhante nos experimentos A (a pleno sol) e B (sob sombrite) para a maioria das variáveis analisadas, embora as plantas cultivadas em B (radiação média de $218 \mu \mathrm{mol} \mathrm{m} \mathrm{m}^{-2} \mathrm{~s}^{-1}$ ) tivessem maior duração do ciclo de desenvolvimento, cerca de 140 DAT, em relação às plantas mantidas sob A (média de $658 \mu \mathrm{mol} \mathrm{m} \mathrm{m}^{-2} \mathrm{~s}^{-1}$ ), com ciclo de aproximadamente 110 DAT. A diferença percentual de radiação entre as duas condições foi, em média, de $67 \%$ ao longo do ciclo.

As plantas sombreadas (B) apresentaram área foliar $\left(\mathrm{A}_{\mathrm{f}}\right)$ de $846 \mathrm{~cm}^{2}$ planta $^{-1}$ durante o ciclo, cerca de três vezes superior à média de $277 \mathrm{~cm}^{2}$ planta ${ }^{-1}$, mantidas a plena luz (A) (Figura 1). A A foi crescente até $58\left(1.503 \mathrm{~cm}^{2}\right.$ planta $\left.^{-1}\right)$ e $40\left(565 \mathrm{~cm}^{2}\right.$ planta $\left.^{-1}\right)$ DAT, para B e $A$, respectivamente, quando se iniciou o processo de senescência foliar. Resultados semelhantes foram obtidos por Vlahos et al. (1991) para três diferentes cultivares de Archimene; por Muroya et al. (1997), em estudo com Calophyllum angulare A.C. Smith; e por Farias et al. (1997), em Cedrelinga catenaeformis (Ducke) Ducke, que verificaram um rápido aumento da superfície fotossintetizante como forma de as plantas assegurarem um aproveitamento maior das baixas intensidades luminosas.

Quanto à altura (Figura 1), medida de dimensão linear, observou-se maior cobertura vegetal nas plantas de $S$. orientalis mantidas sob baixa radiação (B), altura máxima de $45 \mathrm{~cm}$ planta $^{-1}$, obtida aos 91 DAT, e $26 \mathrm{~cm}_{\text {planta }}{ }^{-1}$ aos 75 DAT sob alta radiação (A). Esse mesmo comportamento também foi observado nos 
estudos de Pushpakumari \& Sasidhar (1992) com Discorea alata, D. esculenta e Xanthosoma sangittifolium e por Muroya et al. (1997) com Calophyllum angulare, em que as maiores alturas observadas sob sombreamento, provavelmente, se deveram ao aumento da dominância apical, em decorrência do decréscimo de fotoassimilados e dos maiores níveis de auxina.

A contagem de estruturas morfológicas e os números de folhas e inflorescências (Figura 1) fornecem dados relevantes quanto à fenologia das plantas (Benincasa, 1988). Para ambas as condições de luminosidade, embora já houvesse iniciado o processo de senescência foliar, obteve-se o número máximo de folhas aos $74 \mathrm{e}$ 55 DAT, para B e A, com médias estimadas de
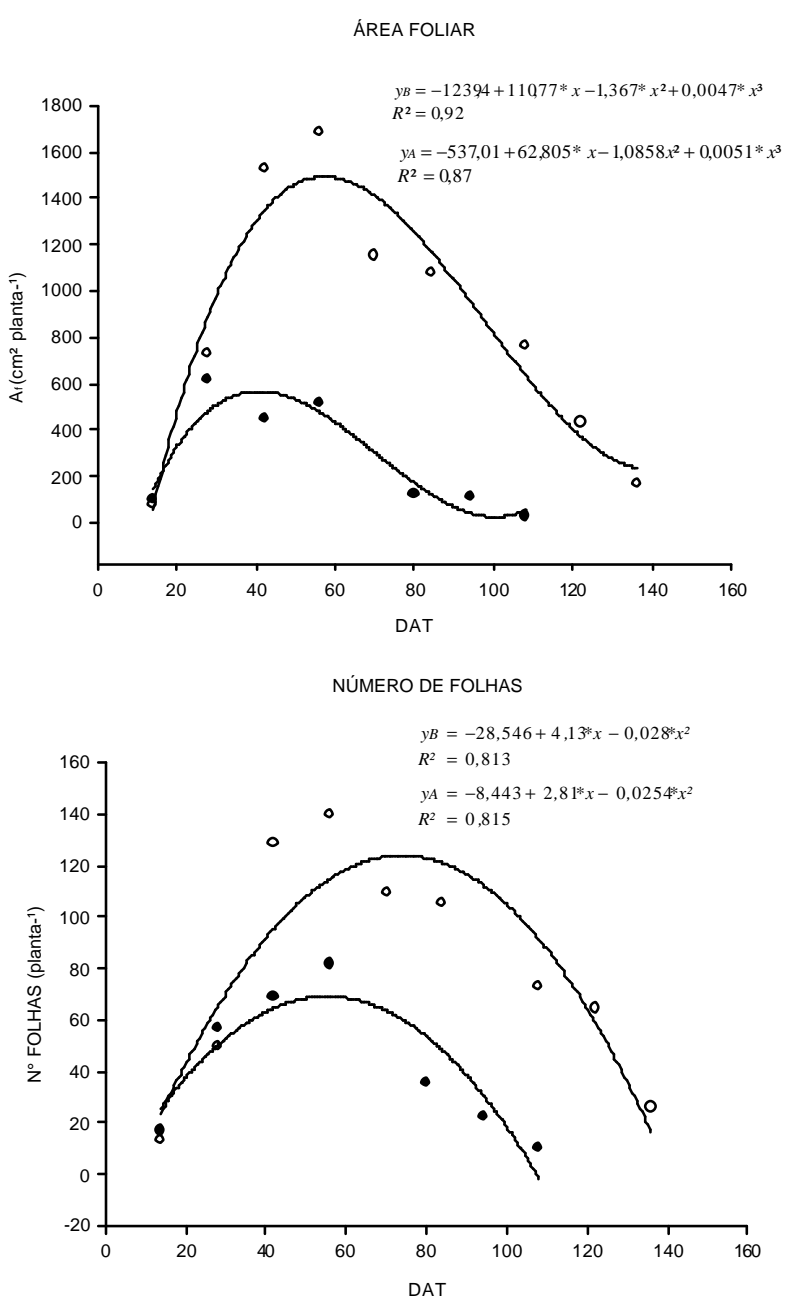

124 e 69 folhas planta $^{-1}$, respectivamente. Quanto ao desenvolvimento reprodutivo, observou-se que houve formação de capítulos florais logo na segunda avaliação, com os pontos máximos estimados aos 89 e 70 DAT, média de 367 e 278 flores planta $^{-1}$, para B e A. Tanto para o número de folhas como para o de flores, as plantas sob sombreamento obtiveram maiores médias e os pontos de máxima estimados mais tardiamente. Vlahos et al. (1991), em experimento com cultivares de Achimenes, também observaram que a diminuição da intensidade luminosa favoreceu o florescimento; esses dados foram contrários aos de Cooper (1967), que verificou decréscimo da produção de flores de Lotus corniculatus e Medicago sativa com a redução da luminosidade.

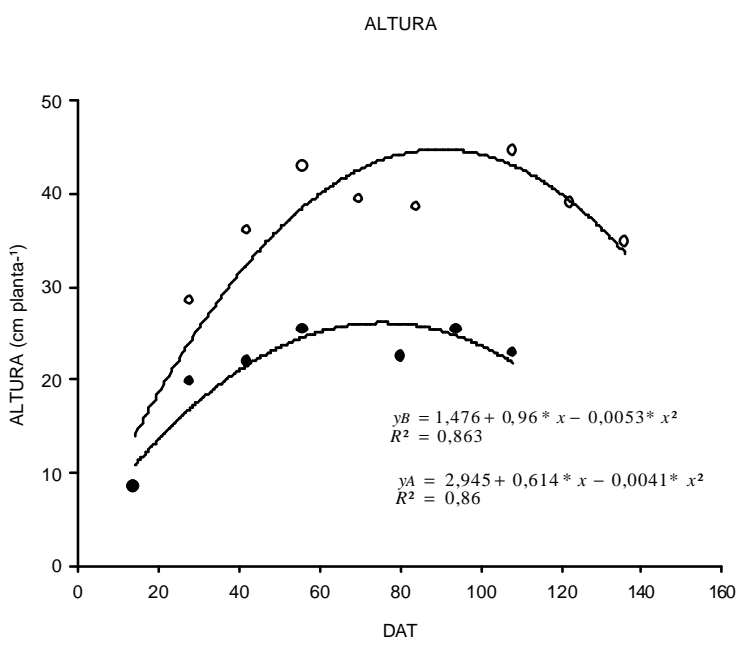

NÚMERO DE CAPÍTULOS

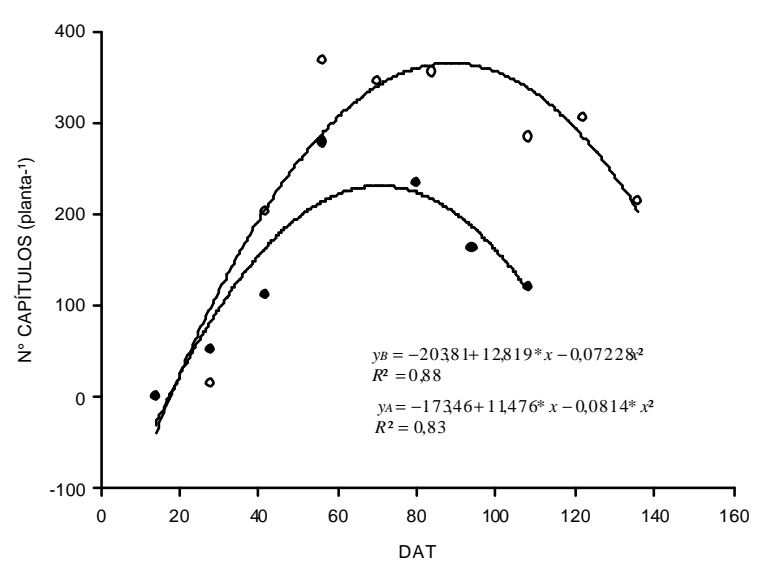

Figura 1 - Área foliar, altura, número de folhas e capítulos florais de Siegesbeckia orientalis ao longo do ciclo de crescimento, sob alta $(\bullet)$ e baixa $(O)$ radiação. 
De acordo com os dados de biomassa seca total $\left(\mathrm{W}_{\mathrm{t}}\right)$ de $\mathrm{S}$. orientalis (Figura 2), foram obtidos os pontos máximos aos 92 e 70 DAT, para as condições $\mathrm{B}\left(17,5 \mathrm{~g}_{\text {planta }}{ }^{-1}\right)$ e A (11,4 $\mathrm{g}$ planta-1). O decréscimo da $\mathrm{W}_{\mathrm{t}}$ ao final do ciclo deveu-se, principalmente, à queda no número de folhas. Outros autores também verificaram que algumas espécies são capazes de maximizar a produção de matéria seca com a diminuição da radiação, como em Achimenes (Vlahos et al., 1991), Rumex obtusifolius (Jeangros \& Nosberger, 1992) e Cynosurus cristatus (Cooley et al., 2000); o estabelecimento de tais espécies não é prevenido pelo sombreamento.

A distribuição de matéria seca nas diferentes partes da planta (Figura 2) se deve ao
MASSA SECA TOTAL

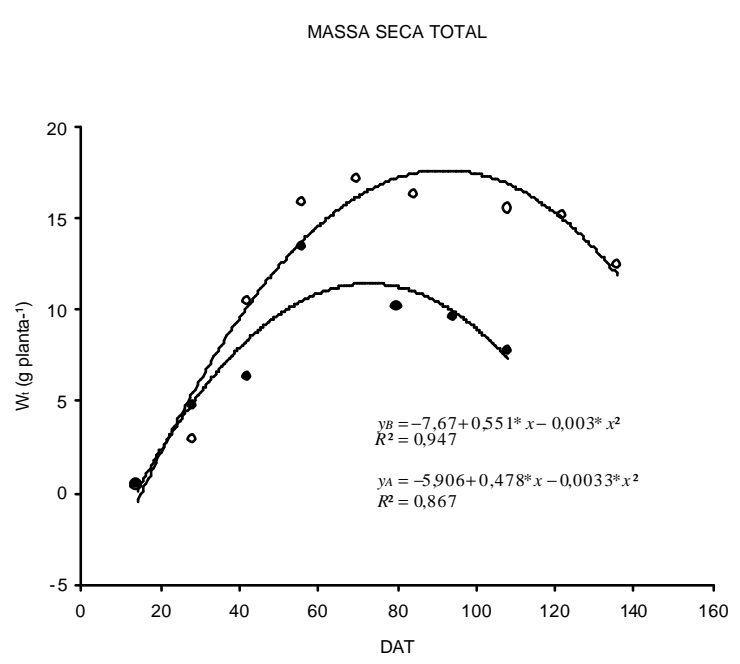

RAZÃO DE MASSA DE PARTE AÉREA

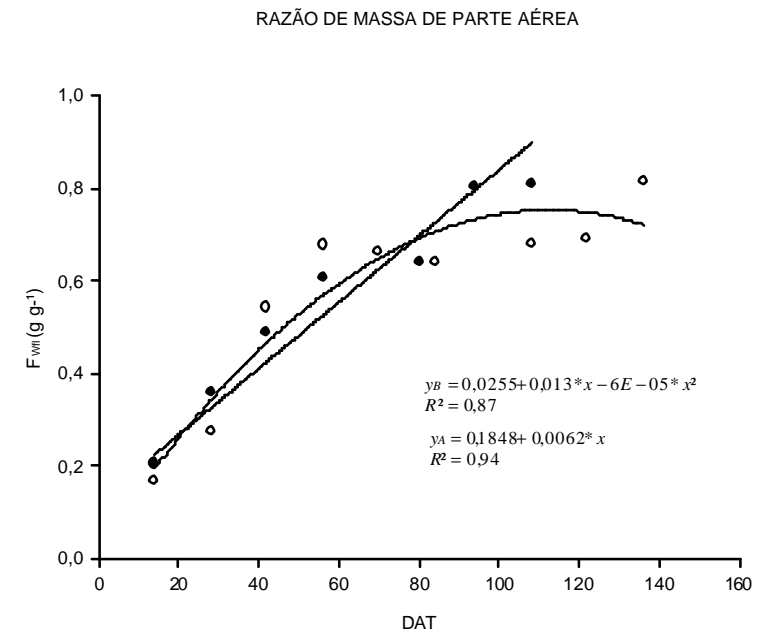

processo fisiológico da translocação de fotoassimilados ao longo do ciclo cultural. Para ambas as condições de radiação, a razão de massa seca nas folhas $\left(\mathrm{F}_{\mathrm{wf}}\right)$ foi decrescente com o ciclo, enquanto a massa seca acumulada na parte aérea $\left(\mathrm{F}_{\mathrm{wf}}\right)$ mostrou-se crescente (Figura 2). No início do ciclo encontram-se valores entre 0,55 e 0,65 para a $\mathrm{F}_{\mathrm{wf}}$, evidenciando que nesta fase 55 a $65 \%$ do total da biomassa encontrava-se nas folhas. Após este período inicial, houve inversão na representatividade das folhas e da parte aérea. Ao final do ciclo, em ambos os experimentos, a biomassa seca acumulada na parte aérea $\left(\mathrm{F}_{\mathrm{wfl}}\right)$ chegou a representar em torno de $80 \%$. Quanto à razão de massa radicular $\left(\mathrm{F}_{\mathrm{wr}}\right)$, estimou-se o mínimo aos 125 DAT para B (0,05 $\left.\mathrm{g} \mathrm{g}^{-1}\right)$ e o máximo aos 36 DAT para A $\left(0,195 \mathrm{~g} \mathrm{~g}^{-1}\right)$.

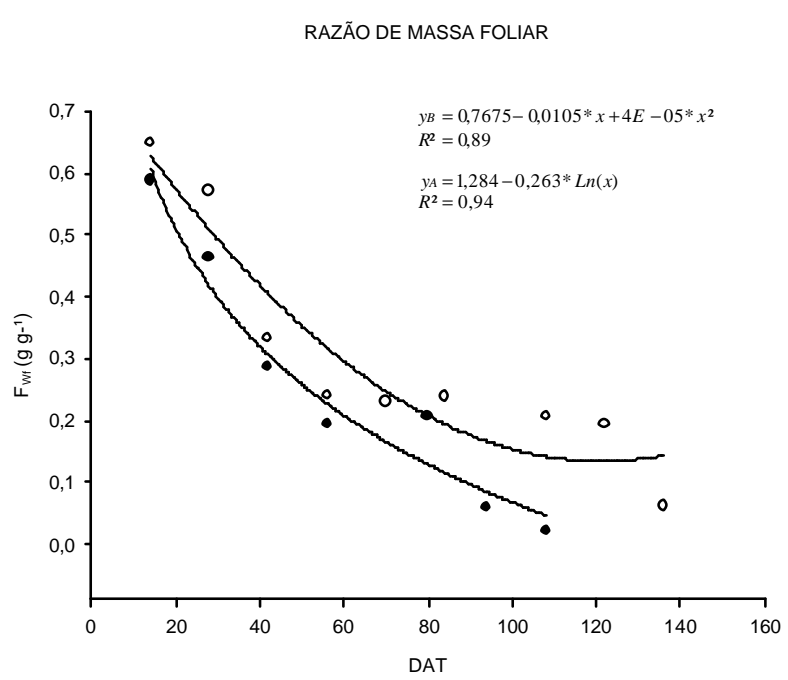

RAZÃO DE MASSA RADICULAR

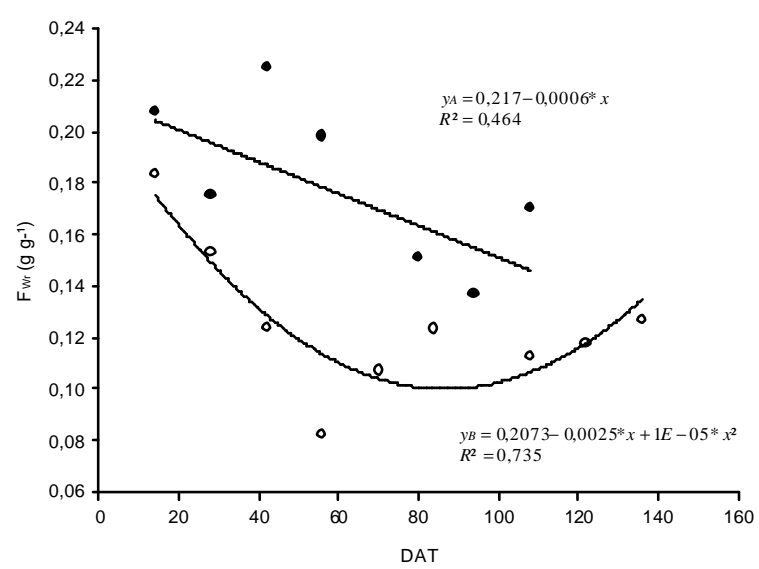

Figura 2 - Massa seca total e a sua distribuição nas diferentes partes de Siegesbeckia orientalis ao longo do ciclo de crescimento, sob alta $(\bullet)$ e baixa $(O)$ radiação. 
Corroborando os trabalhos de Dale \& Causton (1992b,c) com três diferentes espécies de Veronica, Sharma (1994) com Panicum maximum Jacq. e Farias et al. (1997) com Cedrelinga catenaeformis, na condição B, a média da razão de massa foliar $\left(\mathrm{F}_{\mathrm{wf}}\right)$ foi superior durante o ciclo: $0,3 \mathrm{~g} \mathrm{~g}^{-1}$ contra $0,25 \mathrm{~g} \mathrm{~g}^{-1} \mathrm{em} \mathrm{A}$. Entretanto, essa diferença se torna muito pequena, considerando a superioridade de três vezes em área foliar $\left(\mathrm{A}_{\mathrm{f}}\right)$ obtida na condição $\mathrm{B}$, em relação a A. Isso indica que, apesar de apresentarem menor $A_{f}$, as folhas das plantas sob luz plena tiveram maior espessamento do mesofilo e, conseqüentemente, maior massa por superfície de folha. A razão de massa seca de raiz $\left(\mathrm{F}_{\mathrm{wr}}\right)$ e parte aérea $\left(\mathrm{F}_{\mathrm{wfl}}\right)$ foi maior para $\mathrm{o}$ experimento A - média de 18 contra $12 \%$ (B) da matéria seca total para raiz e 56 contra $52 \%$ (B) para parte aérea. Em espécies de Veronica também se observou a diminuição da $\mathrm{F}_{\mathrm{wr}} \mathrm{com}$ o sombreamento; quanto a $\mathrm{F}_{\mathrm{wff}}$, o comportamento foi o oposto (Dale \& Causton, 1992a, b, c).

De modo geral, as plantas de $S$. orientalis apresentaram um rápido crescimento inicial (Figura 3). Para as plantas sob sombreamento, a taxa de crescimento absoluto $\left(\mathrm{C}_{t}\right)$ obteve sua velocidade de incremento máximo ao redor de 40-50 DAT, seguido por redução gradual, até atingir valores negativos, entre 80 e 90 DAT, devido ao processo de senescência da planta. Por outro lado, para as plantas sob alta radiação, a $\mathrm{C}_{\mathrm{t}}$ atingiu valores máximos logo na segunda avaliação: 28 DAT. A partir de 60 DAT, observou-se rápida senescência dos órgãos da planta. Considerando-se somente os valores positivos das $\mathrm{C}_{t}(\mathrm{~s})$, as plantas de $S$. orientalis apresentaram velocidade média de crescimento de 0,2 e 0,3 $\mathrm{g} \mathrm{dia}^{-1}$, para as condições de alta e baixa luminosidade, respectivamente.

Na Figura 4, para ambos os experimentos, as diferentes partes de S. orientalis mostraram comportamento semelhante quanto às taxas de crescimento relativo $\left(\mathrm{R}_{\mathrm{w}}\right)$. Os valores da $\mathrm{R}_{\mathrm{w}}$ foram crescentes até 28 DAT, seguido por decréscimo das taxas até o final do ciclo, devido, principalmente, ao auto-sombreamento. Considerando-se somente as $\mathrm{R}_{\mathrm{w}}(\mathrm{s})$ positivas, em $\mathrm{B}$, os valores médios corresponderam a $0,036 \mathrm{~g} \mathrm{~g}^{-1}$ $\mathrm{dia}^{-1}$ para raiz, 0,055 $\mathrm{g} \mathrm{g}^{-1} \mathrm{dia}^{-1}$ para parte aérea, $0,042 \mathrm{~g} \mathrm{~g}^{-1} \mathrm{dia}^{-1}$ para folhas e 0,057 $\mathrm{g} \mathrm{g}^{-1} \mathrm{dia}^{-1}$ total. Para plantas sob A, os valores estimados para as $R_{\mathrm{w}}(\mathrm{s})$ foram superiores aos da

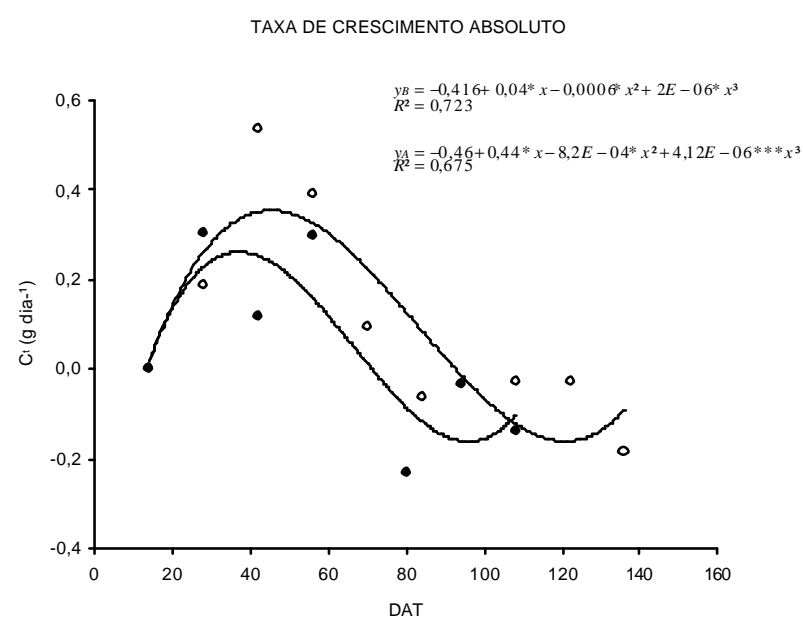

Figura 3 - Taxa de crescimento absoluto de Siegesbeckia orientalis ao longo do ciclo de crescimento, sob alta $(\bullet)$ e baixa $(O)$ radiação.

condição anterior: $0,054 \mathrm{~g} \mathrm{~g}^{-1} \mathrm{dia}^{-1}$ para raiz, $0,06 \mathrm{~g} \mathrm{~g}^{-1}$ dia $^{-1}$ para parte aérea e $0,056 \mathrm{~g} \mathrm{~g}^{-1}$ dia $^{-1}$ para folhas. No entanto, quando se observa a $\mathrm{R}_{\mathrm{w}}$ total, o índice foi um pouco inferior: $0,05 \mathrm{~g} \mathrm{~g}^{-1} \mathrm{dia}^{-1}$. Isso ocorre porque $\mathrm{A}_{\mathrm{w}}$ é dependente de dois outros parâmetros de crescimento: a área foliar útil para o crescimento $\left(\mathrm{F}_{\mathrm{A}}\right)$ e a taxa de fotossintese líquida $\left(\mathrm{E}_{\mathrm{A}}\right)$ (Cooper, 1967; Benincasa, 1988). Esses resultados concordam com os trabalhos de Cooper (1967) com Lotus corniculatus e Medicago sativa, Jeangros \& Nosberger (1992) com Rumex obtusifolius e Lolium perene L. e Dale \& Causton (1992a, b, c) com espécies de Veronica.

De acordo com Radford (1967) e Benincasa (1988), a fórmula da taxa de assimilação líquida $\left(\mathrm{E}_{\mathrm{A}}\right)$ somente se aplica quando existe correlação linear entre área foliar e matéria seca total. Uma vez que não foi encontrada tal correlação para nenhuma das situações estudadas, esses dados não foram apresentados em forma de regressão. Entretanto, considerando-se somente as taxas positivas, a $\mathrm{E}_{\mathrm{A}}$ foi de 0,00025 e 0,0005 $\mathrm{g} \mathrm{dia}^{-1} \mathrm{~cm}^{2}$, para as condições B e A, respectivamente, sendo o principal fato para explicar as maiores $\mathrm{R}_{\mathrm{w}}(\mathrm{s})$ na condição $\mathrm{A}$, em que as plantas de $S$. orientalis produziram maior biomassa por área foliar, demonstrando menor competitividade da espécie sob alta radiação incidente.

A razão de área foliar $\left(\mathrm{F}_{\mathrm{A}}\right)$ é um componente morfofisiológico que expressa a área foliar útil para a fotossintese. Na Figura 5, verifica-se que 
TAXA DE CRESCIMENTO RELATIVO TOTAL

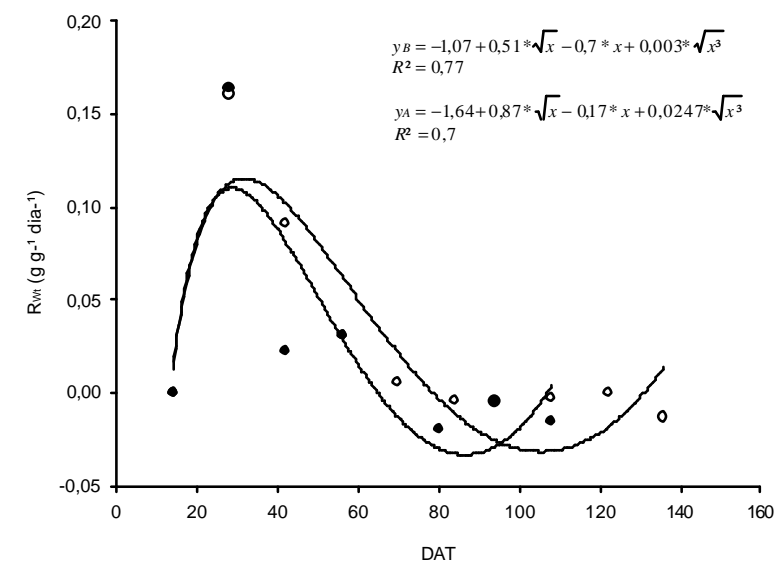

TAXA DE CRESCIMENTO RELATIVO DA PARTE AÉREA

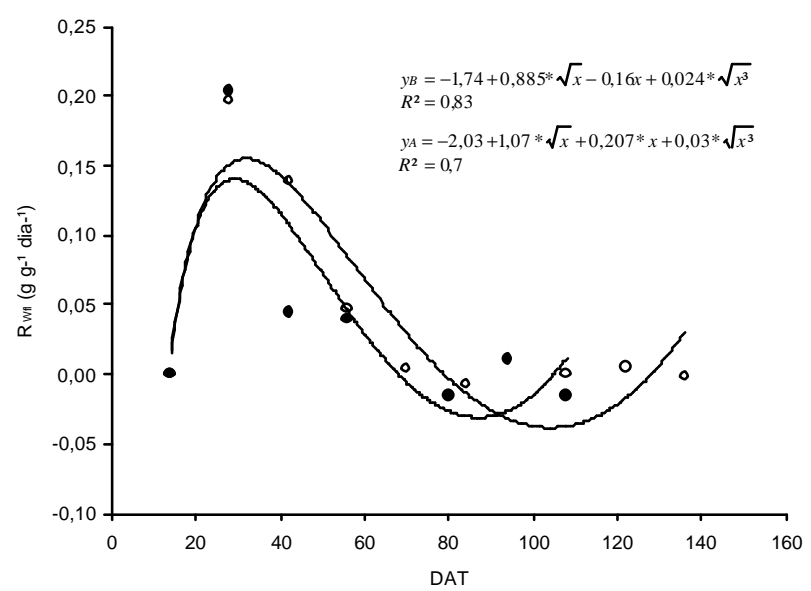

TAXA DE CRESCIMENTO RELATIVO DE FOLHAS

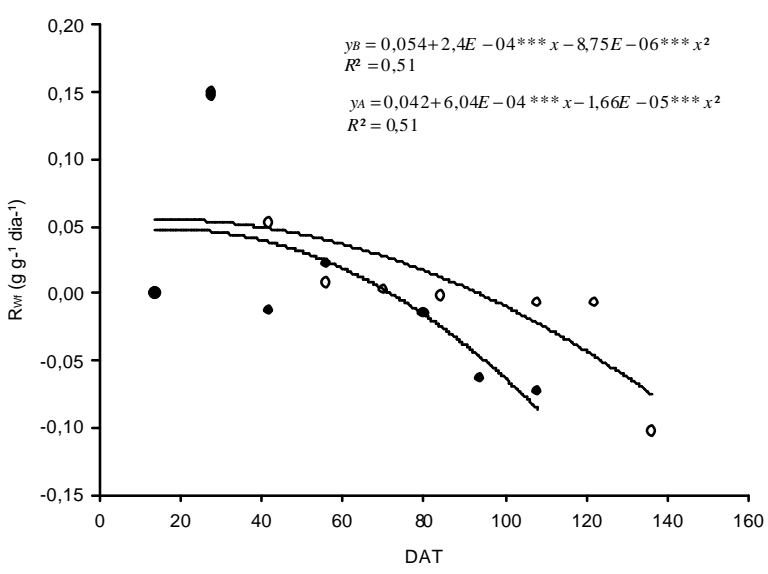

TAXA DE CRESCIMENTO RELATIVO DE RAIZ

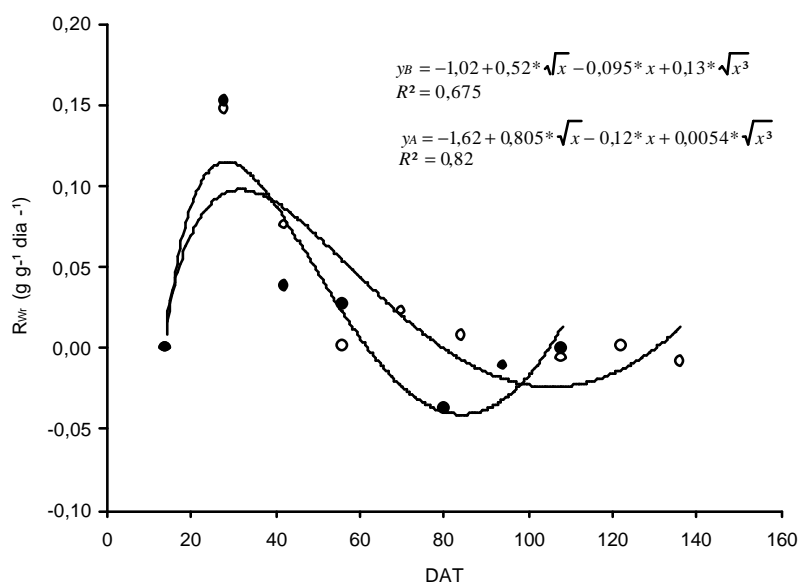

Figura 4 - Taxa de crescimento relativo nas diferentes partes de Siegesbeckia orientalis ao longo do ciclo de crescimento, sob alta $(\bullet)$ e baixa $(O)$ radiação.

RAZÃO DE ÁREA FOLIAR

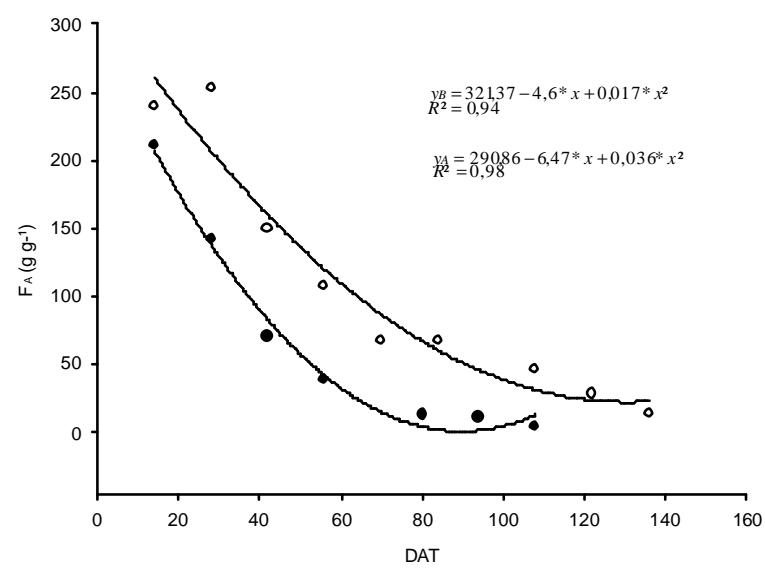

ÁREA FOLIAR ESPECÍFICA

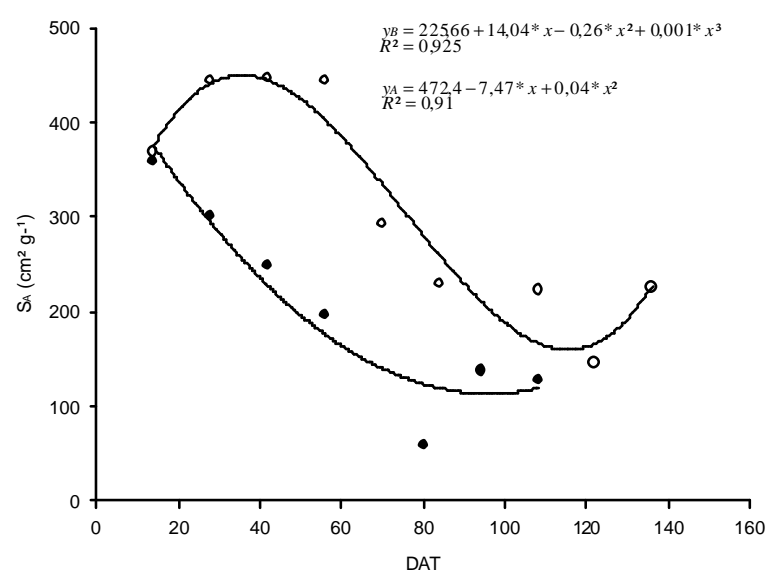

Figura 5 - Razão de área foliar e área foliar específica de Siegesbeckia orientalis ao longo do ciclo de crescimento, sob alta $(\bullet)$ e baixa $(O)$ radiação. 
a $\mathrm{F}_{\mathrm{A}}$ foi maior para as plantas na condição $\mathrm{B}$ média durante o ciclo de 108 contra $69 \mathrm{~cm}^{2} \mathrm{~g}^{-1}$ para $\mathrm{A}-$, indicando que S. orientalis é favorecida por ambientes sombreados, pois as plantas consideradas de sombra, geralmente, apresentam baixa $\mathrm{F}_{\mathrm{A}}$ a pleno sol, como resultado da capacidade da planta em se adaptar a diferentes condições de luminosidade, assim como nas espécies estudadas por Cooper (1967), Dale \& Causton (1992a, b, c), Sharma (1994) e Farias et al. (1997). Esses dados permitem também verificar que a $F_{A}$ declina à medida que a planta cresce, pois, com o crescimento, aumenta a interferência devido ao auto-sombreamento e diminui a área foliar.

A área foliar específica $\left(S_{A}\right)$ é o componente da $F_{A}$ que relaciona a superfície foliar (parte morfológica) com o peso de matéria seca da própria folha (parte anatômica). O inverso da $\mathrm{S}_{\mathrm{A}}$ indica a espessura da folha. $\mathrm{A} \mathrm{S}_{\mathrm{A}}$ foi superior para a condição $B$ - média de 313 contra $204 \mathrm{~cm}^{2} \mathrm{~g}^{-1}$ em A (Figura 5) -, indicando que as plantas de $S$. orientalis apresentam maior espessamento das folhas quando submetidas à alta radiação, corroborando os experimentos de Sharma (1994) e Dale \& Causton (1992a, b).

De maneira geral, conclui-se que as plantas de $S$. orientalis iniciaram sua fase reprodutiva precocemente, apresentaram plasticidade e se adaptaram a diferentes condições de luminosidade. Sob menor radiação incidente, entretanto, as plantas tiveram seu ciclo prolongado em torno de 30 dias, retardando os valores máximos e/ou mínimos. Obtiveram, também, as maiores médias para a maioria dos índices avaliados $\mathrm{A}_{\mathrm{f}}$, altura, números de folhas e capítulos florais, $\mathrm{W}_{\mathrm{t}}, \mathrm{C}_{\mathrm{t}}, \mathrm{R}_{\mathrm{w}}, \mathrm{F}_{\mathrm{w}}, \mathrm{F}_{\mathrm{A}}$ e $\mathrm{S}_{\mathrm{A}}-$, sugerindo, assim, que a espécie é favorecida pelo sombreamento.

\section{LITERATURA CITADA}

BARUAH, R. N. et al. A new melampolide from Siegesbeckia orientalis. Phytochemistry, v. 18, p. 991-994, 1979.

BARUAH, R. N. et al. New melampolides and darutigenol from Siegesbeckia orientalis. Phytochemistry, v. 19, p. 323325, 1980.

BENINCASA, M. M. P. Análise de crescimento de plantas, noções básicas. Jaboticabal: FUNEP, 1988. 42 p.

BOHLMANN, F. et al. Neue melampolide und cis, cisgermacranolide aus vertretern der subtribus Melampodiinae. Phytochemistry, v. 18, p. 625-630, 1979.
BREMER, K. Asteraceae, cladistics \& classification. Portland: Timber Press, 1994. 752 p.

COOLEY, N. M. et al. Outdoor ultraviolet polychromatic action spectra for growth responses of Bellis perennis and Cynosurus cristatus. J. Photochem. Photobiol. Biol., v. 59, p. 64-71, 2000.

COOPER, C. S. Relative growth of alfafa and birdsfoot treafoil seedlings under low light intensity. Crop Sci., v. 7, n. 3, p.176-178, 1967.

DALE, M. P.; CAUSTON, D. R. The ecophysiology of Veronica chamaedrys, V. montana and V. officinalis. I. Light quality and light quantity. J. Ecol., v. 80, p. 483-492, 1992a.

DALE, M. P.; CAUSTON, D. R. The ecophysiology of Veronica chamaedrys, V. montana and V. officinalis. II. The interaction of irradiance and water regime. J. Ecol., v. 80, p. 493-504, 1992 b.

DALE, M. P.; CAUSTON, D. R. The ecophysiology of Veronica chamaedrys, V. montana and V. officinalis. III. Effects of shading on the phenology of biomass allocations - a field experiment. J. Ecol., v. 80, p. 505-515, 1992 c.

FARIAS, V. C. C. et al. Análise de crescimento de mudas de cedrorana (Cedrelinga catenaeformis (Ducke) Ducke) cultivadas em condições de viveiro. R. Bras. Sementes, v. 19, n. 2, p. 193-200, 1997.

HEINRICH, G. et al. Glandular hairs of Siegebeckia jorullensis Kunth (Asteraceae): morphology, histochemistry and composition of essential oil. Ann. Bot., v. 89, p. 459469, 2002.

JEANGROS, B.; NOSBERGER, J. Comparison of the growth response of Rumes obtusifolius L. and Lolium perenne L. to photon flux density. Weed Res. Oxford, v. 32, n. 4, p. 311-316, 1992.

KISSMANN, K. G.; GROTH, D. Plantas infestantes e nocivas. São Paulo: BASF Brasileira, 1992. p. 326-328. T. 2 .

MUROYA, K.; VARELA, V. P.; CAMPOS, M. A. A. Análise de crescimento de mudas de jacareúba (Calophyllum angulare A.C. Smith - Guttiferae) cultivadas em condições de viveiro. Acta Am., v. 27, n. 3, p. 197-212, 1997.

MUSIL, C. F.; CHIMPHANGO, S. B. M.; DAKORA, F. D. Effects of elevated ultraviole-B radiation on native and cultivated plants of southern Africa. Ann. Bot., v. 90, p. 127-137, 2002.

PUDLES, J.; DIARA, A.; LEDERER, E. Sur l'isolement et la constitution chimique du darutoside, principe amer de Siegesbeckia orientalis. Comptes Rendus Herbd. Sea. l'academie Sci., v. 244, n. 4, p. 472-475, 1957. 
PUSHPAKUMARI, R.; SASIDHAR, V. K. Influence of shade on the growth attributes of minor tuber crops. J Root. Crops, v. 18, n. 1, p. 64-67, 1992.

RADFORD, P. J. Growth analysis formulae. Their use and abuse. Crop Sci., v. 7, n. 3, p. 171-175, 1967.

REIS, M. S.; MARIOT, A. Diversidade natural e aspectos agronômicos de plantas medicinais. In: SIMÕES, C. M. O. et al. Farmacognosia: da planta ao medicamento. 4.ed. Florianópolis: Universidade Federal de Santa Catarina, 2002. p. 41-62.
SHARMA, B. M. Ecophysiological studies on Panicum maximum Jacq. In south-west Nigeria. Range Manag. Agrof., v. 15, n. 1, p. 49-53, 1994.

VLAHOS, J. C.; HEUVELINK, E;. MARTAKIS, G. F. P. A growth analysis study of three Achimenes cultivars grown under three light regimes. Sci. Hort., v. 46, n. 3-4, p. $275-$ 282, 1991.

ZDERO, C. et al. Sesquiterpene lactones and other constituents from Siegesbeckia orientalis and Guizotia scabra. Phytochemistry, v. 30, n. 5, p. 1579-1584, 1991. 